\title{
All-Atom Molecular Dynamics of Virus Capsids as Drug Targets
}

\section{Supplemental Information}

Juan R. Perilla, ${ }^{*, \dagger}$, Jodi A. Hadden, $^{\dagger}$ Boon Chong Goh, ${ }^{\dagger, \ddagger}$ Christopher G. Mayne $^{\dagger}$ and Klaus Schulten*,†,

$\dagger$ Beckman Institute, University of Illinois at Urbana-Champaign, Urbana, Illinois 61801, United States

$\ddagger$ Department of Physics, University of Illinois at Urbana-Champaign, Urbana, Illinois 61801, United States

E-mail: jperilla@illinois.edu; kschulte@ks.uiuc.edu 


\section{Parameterizing small-molecule drugs}

Simulations of virus capsids complexed to small molecule drugs, HAP1 and PF74, requires force field parameters that are specifically tailored to describe the internal dynamics of these molecules and their interactions with the surrounding environment (i.e., solvent, ions, protein). The Force Field Toolkit (ffTK) ${ }^{1}$ plugin in $\mathrm{VMD}^{2}$ was specifically designed to optimize parameters describing small molecules in a manner that is compatible with the CHARMM family of force fields, most notably the popular CHARMM36 force field for proteins. ${ }^{3,4}$ Following the parametrization philosophy outlined for the CHARMM General Force Field $(\mathrm{CGenFF}),{ }^{5}$ ffTK provides a graphical user interface that facilitates fitting molecular mechanical force field parameters against high level quantum mechanical (QM) target data, and includes analytical tools to aid in assessing the progress of the optimization routines and quality of the final fits.

HAP1. HAP1 is a dihydropyrimidine core bearing methyl, methyl ester, pyridyl, and 2-chloro4-fluorophenyl substituents. The standard CGenFF distribution offers the parameters of individual chemical groups, ${ }^{5}$ but lacks the linkages (bonds, angles and dihedrals) connecting these substructures. The CGenFF program ${ }^{6,7}$ (formerly known as ParamChem) was used to identify missing parameters and assign atom types and partial atomic charges by analogy to residues provided in CGenFF. The missing bond, angle and dihedral parameters (Figure S1A), and the partial charges of all atoms were optimized using ffTK. ${ }^{1}$ HAP1, which contains 40 atoms, can be directly optimized as a whole molecule, namely without requiring fragmentation to reduce the system size. The energy profile of the fitted dihedral parameters shows good agreement with the profile computed at the QM level of theory (Figure S1B).

PF74. The PF74 ligand is largely comprised of common structural subunits, e.g., indole, phenylalanine, aniline, for which parameters are available in the standard CGenFF distribution. ${ }^{5}$ Several linkages connecting these substructures, however, are not well described and required parameterization using ffTK. All atom types were manually assigned by analogy to residues in the CGenFF parameter set. Based on the type assignments, each parameter required to describe PF74 was crosschecked against CGenFF to identify all terms that were missing and required parametriztion. The majority of charges were assigned by analogy to existing charge groups, with the exception of the $N, N$-methylphenyl amide moiety, which was optimized directly in ffTK. PF74 is larger than HAP1, in 
fact too large to be described quantum chemically as a whole. In such cases, a "divide-and-conquer" strategy is the best approach to mitigate computational overhead and to minimize technical problems due to the three-dimensional topology of some molecules (Figure S2A). The details of this process are tedious and largely technical, but in short, the PF74 ligand was fragmented into representative model systems encompassing all missing charges and parameters (Figure S2B). Within each fragment, all missing charges and parameters were optimized using standard ffTK protocols and recombined to yield a complete parameter set for PF74 (Figure S2C).

\section{Simulations of HBV capsids}

Computational modeling of an HAP1-bound capsid. An all-atom model of the complete HAP1bound HBV capsid was prepared based on the available crystal structure ${ }^{8}$ and corresponding unpublished drug coordinates provided by A. Zlotnick from Indiana University. The protomer chain terminals were built up to match the lengths resolved in the analogous HBV apo-capsid structure, ${ }^{8}$ employing the Model/Refine Loops feature of MODELLER, ${ }^{9}$ as implemented in Chimera-1.10.1. ${ }^{10}$ Ten models were generated for each terminal extension, and the structure with the most favorable energy score that did not produce overlaps with neighboring chains or their respective terminal extensions were selected for the final capsid model. Protonation states were assigned to both the apo-capsid and HAP1-bound capsid using propKa-3.1 $1^{11}$ for an environmental $\mathrm{pH}$ of 7 . The Cionize plugin of VMD $1.9 .2^{2}$ was used to place $\mathrm{Na}^{+}$and $\mathrm{Cl}^{-}$ions around the capsids, according to the local electrostatic potential. Capsids were then suspended in $39.2 \mathrm{~nm}^{3}$ boxes of explicit solvent and bulk ions, taking into account previously placed ions, to produce a charge-neutral system in a solution of $150 \mathrm{mM} \mathrm{NaCl}$. Simulation files describing the capsid systems were prepared using the psfgen plugin in VMD $1.9 .2,{ }^{2}$ applying the CHARMM36 ${ }^{3,4}$ force field, CHARMM TIP3P ${ }^{12}$ water model, and custom CHARMM-compatible drug parameters developed specifically for HAP1.

Simulation protocol. All simulations were performed with NAMD $2.10^{13}$ on the NCSA Blue Waters supercomputer. A steepest decent energy minimization protocol consisting of 30,000 cycles was applied to minimize, first the solvent, then the side chains of the capsid systems. Upon initiating dynamics, the temperature of each system was gradually increased from $50 \mathrm{~K}$ to $300 \mathrm{~K}$ over an interval of $5 \mathrm{~ns}$, applying Cartesian restraints of $5 \mathrm{kcal} / \mathrm{mol}$ to the protein backbone. Continuing under isothermal, 
isobaric conditions, backbone restraints were gradually removed over an additional interval of $5 \mathrm{~ns}$. An arbitrary equilibration time of 5 ns was granted, during which quaternary structure began to relax from its crystallographic conformation. Production simulations totaling 100 ns were collected for both capsid systems at a timestep of $1 \mathrm{fs}$, saving frames every $5 \mathrm{ps}$.

Temperature regulation was performed with the Langevin thermostat algorithm in NAMD, employing a damping coefficient of $1 \mathrm{ps}^{-1}$. The Nosé-Hoover Langevin piston control was applied to maintain constant pressure of 1 bar, allowing isotropic cell scaling, with piston oscillation period of $2000 \mathrm{fs}$ and damping timescale of $1000 \mathrm{fs}$. All bonds to hydrogen were constrained with the SHAKE (solute) or SETTLE (solvent) algorithms. Long-range electrostatics were split from short-range at a cutoff of $1.2 \mathrm{~nm}$ according to a quintic polynomial splitting function and computed with the PME (particlemesh Ewald) method, as implemented in NAMD. Full electrostatic evaluations were performed every two time steps.

\section{Simulations of HIV-1 pentamer-of-hexamers}

Computational modeling of a PF74-bound pentamer of hexamers. Structure and trajectories for the apo-form of the pentamer of hexamers $(\mathrm{POH})$ were taken from a previous publication. ${ }^{14}$ In order to derive a model of the $\mathrm{POH}$ in complex with PF74, coordinates for the drug were obtained from the X-ray structure of hexameric CA. ${ }^{15}$ The RMSD for the $C_{\alpha}$ atoms of the N-terminal domain (NTD), between PF74-bound hexamer and the NTDs of the apo-form POH was 1.4 $\AA$, which permitted docking of PF74 into the POH by rms-fitting the PF74-bound hexamer to each of the NTDs in the POH. Any steric clashes between docked PF74 molecules and water or ions in the simulation box were addressed by removing the offending water and ions. The total charge of the system was neutralized by replacing water molecules in the bulk by charged ions.

Simulation protocol. All simulations carried out for the POH-PF74 complex used the r-RESPA integrator available in NAMD. Long-range electrostatic force calculations employed the PME (particlemesh Ewald) method, utilizing a grid spacing of 2.1 Åand 8th order interpolation, with a $1.2 \mathrm{~nm}$ cutoff. Simulations using the CHARMM additive force field, employed a time-step of $2 \mathrm{fs}$, with non-bonded interactions evaluated every 2 fs and electrostatics updated every 4 fs. All hydrogen bonds were constrained with the SHAKE algorithm. Simulations of PF74-bound POH were performed using 
NAMD2 $.9,{ }^{13}$ with the CHARMM $36^{3,4}$ parameters for proteins, CHARMM TIP3P ${ }^{12}$ water model, and custom CHARMM-compatible drug parameters developed specifically for PF74, as described above. Equilibration used Cartesian restraints of $5 \mathrm{kcal} / \mathrm{mol}$ on the protein backbone and first solvation shell, allowing PF74 relaxation within the binding pocket. Continuing under isothermal, isobaric conditions, backbone restraints were gradually removed over an additional interval of 5 ns. Production simulations totaling $1.25 \mu$ s were performed on the NSF Blue Waters supercomputer.

Network Analysis. Dynamical network analysis is commonly applied to map allosteric pathways in proteins, ${ }^{16,17}$ as well as the effects of ligand binding on such pathways. ${ }^{18,19}$ To probe the dynamic structure of CA, network models of the apo-form and PF74-bound POHs were constructed based on MD simulations as follows. In each network model, a node was assigned to each protein residue; for the PF74-bound CA, additional nodes were assigned to represent the major functional groups of PF74. Namely, one node was assigned to each phenyl- and indole-group of the drug (Figure S2A). Edges defining connectivities within the network were drawn for nodes that fell within a cutoff distance of $4.5 \AA$ for at least $75 \%$ of the simulation trajectory. Then, edges between nodes $i$ and $j$ were assigned a weight $w_{i, j}=-\log \left(\left|C_{i, j}\right|\right)$, where $C_{i, j}$ are the Pearson correlation coefficients between nodes $i$ and $j$, given by $C_{i, j}=\frac{\left\langle\Delta \vec{r}_{i}(t) \cdot \overrightarrow{r_{j}}(t)\right\rangle}{\left(\left\langle\Delta \vec{r}_{i}(t)^{2}\right\rangle\left\langle\Delta \vec{r}_{j}(t)^{2}\right\rangle\right)^{\frac{1}{2}}}, \Delta \vec{r}_{i}(t)=\vec{r}_{i}(t)-\left\langle\vec{r}_{i}(t)\right\rangle$, and $\vec{r}_{i}(t)$ is the position of node $i .{ }^{16}$ The resulting network models describing the apo-form and PF74-bound CA systems contain information about the correlation between motions in all parts of the CA protein; thus permitting the determination of highly-correlated paths of residues that connect any two regions of CA. The dynamic correlation between the CypA-binding site and CA-hinge was extracted from the network models created for both apo-form and PF74-bound CA using the NetworkAnalysis ${ }^{16,20}$ tool available in VMD. ${ }^{2}$ The optimal pathways linking the CypA-binding loop and CA-hinge were projected onto the underlying molecular structure (Figure 4D). 
A

bonds

angles

dihedrals<smiles>COC(=O)C1=C(C)NC(c2ccccn2)=N[C@H]1c1ccc(F)cc1Cl</smiles><smiles></smiles><smiles>COC(=O)C1=C(C)NC(c2ccccn2)=N[C@H]1c1ccc(F)cc1Cl</smiles>

B



Figure S1: Parameterization of HAP1 using ffTK. (A) The missing parameters for HAP1 identified using CGenFF program are labeled in red. (B) Fitting of the dihedral parameters, which is the last step of parameterization, shows an accurate reproduction of the torsional potential energy surface when compared against the profile computed at the QM level of theory. 


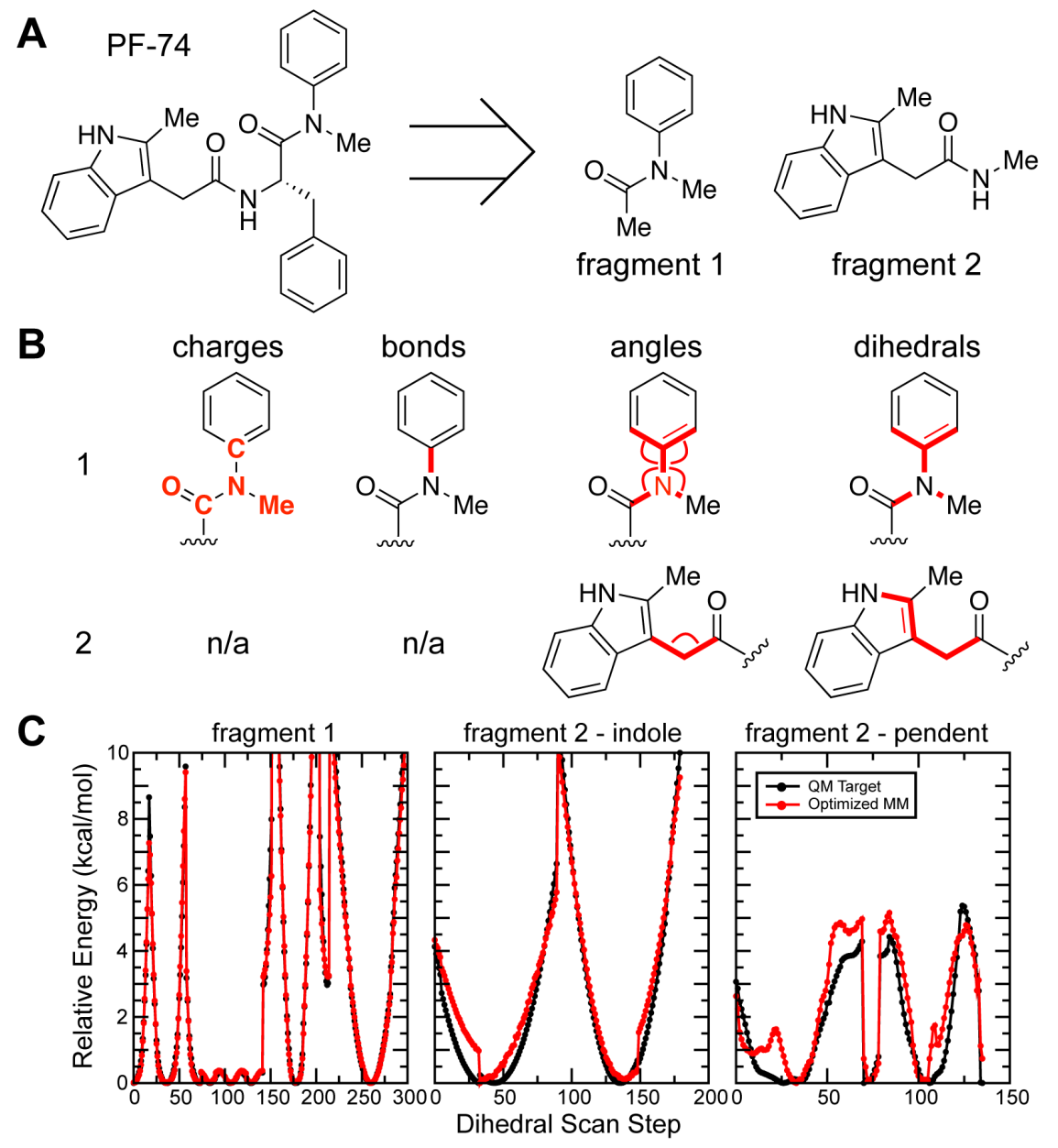

Figure S2: Parameterization of PF-74 using ffTK. (A) PF-74 was fragmented into two model compounds in order to (B) isolate the missing parameters and reduce system size when performing high level QM calculations. (C) Fitting of the dihedral parameters, the last stage of parameterization, shows an accurate reproduction of the torsional potential energy surface when compared against the profile computed at the QM level of theory. 


\section{References}

(1) Mayne, C. G.; Saam, J.; Schulten, K.; Tajkhorshid, E.; Gumbart, J. C. Rapid Parameterization of Small Molecules Using the Force Field Toolkit. Journal of Computational Chemistry 2013, 34, 2757-2770.

(2) Humphrey, W.; Dalke, A.; Schulten, K. VMD - Visual Molecular Dynamics. Journal of Molecular Graphics 1996, 14, 33-38.

(3) MacKerell, Jr., A. D.; Bashford, D.; Bellott, M.; Dunbrack, Jr., R. L.; Evanseck, J. D.; Field, M. J.; Fischer, S.; Gao, J.; Guo, H.; Ha, S. et al. All-Atom Empirical Potential for Molecular Modeling and Dynamics Studies of Proteins. Journal of Physical Chemistry B 1998, 102, 3586-3616.

(4) MacKerell, Jr., A. D.; Feig, M.; Brooks, III, C. L. Improved Treatment of the Protein Backbone in Empirical Force Fields. Journal of the American Chemical Society 2004, 126, 698-699.

(5) Vanommeslaeghe, K.; Hatcher, E.; Acharya, C.; Kundu, S.; Zhong, S.; Shim, J.; Darian, E.; Guvench, O.; Lopes, P.; Vorobyov, I. et al. CHARMM General Force Field: A Force Field for Drug-Like Molecules Compatible with the CHARMM All-Atom Additive Biological Force Fields. Journal of Computational Chemistry 2010, 31, 671-690.

(6) Vanommeslaeghe, K.; MacKerell, Jr., A. D. Automation of the CHARMM General Force Field (CGenFF) I: Bond Perception and Atom Typing. Journal of Chemical Information and Modeling 2012, 52, 3144-3154.

(7) Vanommeslaeghe, K.; Raman, E. P.; MacKerell, Jr., A. D. Automation of the CHARMM General Force Field (CGenFF) II: Assignment of Bonded Parameters and Partial Atomic Charges. Journal of Chemical Information and Modeling 2012, 52, 3155-3168.

(8) Bourne, C. R.; Finn, M. G.; Zlotnick, A. Global Structural Changes in Hepatitis B Capsids Induced by the Assembly Effector HAP1. Journal of Virology 2006, 80, 11055-11061. 
(9) Sali, A.; Blundell, T. L. Comparative Protein Modelling by Satisfaction of Spatial Restraints. Journal of Molecular Biology 1993, 234, 779.

(10) Pettersen, E. F.; Goddard, T. D.; Huang, C. C.; Couch, G. S.; Greenblatt, D. M.; Meng, E. C.; Ferrin, T. E. UCSF Chimera - A Visualization System for Exploratory Research and Analysis. Journal of Computational Chemistry 2004, 25, 1605-1612.

(11) Søndergaard, C. R.; Olsson, M. H.; Rostkowski, M.; Jensen, J. H. Improved Treatment of Ligands and Coupling Effects in Empirical Calculation and Rationalization of pKa Values. Journal of Chemical Theory and Computation 2011, 7, 2284-2295.

(12) Jorgensen, W.; Chandrasekhar, J.; Maudura, J. D.; Impey, R. W.; Klein, M. L. Comparison of Simple Potential Functions for Simulating Liquid Water. Journal of Chemical Physics 1983, 79, 926-935.

(13) Phillips, J. C.; Braun, R.; Wang, W.; Gumbart, J.; Tajkhorshid, E.; Villa, E.; Chipot, C.; Skeel, R. D.; Kale, L.; Schulten, K. Scalable Molecular Dynamics with NAMD. Journal of Computational Chemistry 2005, 26, 1781-1802.

(14) Zhao, G.; Perilla, J. R.; Yufenyuy, E. L.; Meng, X.; Chen, B.; Ning, J.; Ahn, J.; Gronenborn, A. M.; Schulten, K.; Aiken, C. et al. Mature HIV-1 Capsid Structure by Cryo-Electron Microscopy and All-Atom Molecular Dynamics. Nature 2013, 497, 643-646.

(15) Price, A. J.; Jacques, D. A.; McEwan, W. A.; Fletcher, A. J.; Essig, S.; Chin, J. W.; Halambage, U. D.; Aiken, C.; James, L. C. Host Cofactors and Pharmacologic Ligands Share an Essential Interface in HIV-1 Capsid that is Lost upon Disassembly. PLoS Pathogens 2014, 10, e1004459.

(16) Sethi, A.; Eargle, J.; Black, A. A.; Luthey-Schulten, Z. Dynamical Networks in tRNA:Protein Complexes. Proceedings of the National Academy of Sciences of the United States of America 2009, 106, 6620-6625.

(17) Feher, V. A.; Durrant, J. D.; Van Wart, A. T.; Amaro, R. E. Computational Approaches to Mapping Allosteric Pathways. Current Opinion in Structural Biology 2014, 25, 98-103. 
(18) Decherchi, S.; Berteotti, A.; Bottegoni, G.; Rocchia, W.; Cavalli, A. The Ligand Binding Mechanism to Purine Nucleoside Phosphorylase Elucidated via Molecular Dynamics and Machine Learning. Nature Communications 2015, 6, 6155.

(19) Guo, J.; Pang, X.; Zhou, H.-X. Two Pathways Mediate Interdomain Allosteric Regulation in Pin1. Structure 2015, 23, 237-247.

(20) Eargle, J.; Luthey-Schulten, Z. NetworkView: 3D Display and Analysis of Protein- RNA Interaction Networks. Bioinformatics 2012, 28, 3000-3001. 